\title{
Microscopic Manifestations of the Wave Nature and the Fifth Fundamental Field
}

\author{
Louis-Marie Moukala \\ Department of Exact Sciences, Higher Normal School, Brazzaville, Congo \\ Email address: \\ lmmouk@hotmail.fr
}

To cite this article:

Louis-Marie Moukala. Microscopic Manifestations of the Wave Nature and the Fifth Fundamental Field. International Journal of Applied Mathematics and Theoretical Physics. Vol. 5, No. 4, 2019, pp. 104-110. doi: 10.11648/j.ijamtp.20190504.12

Received: November 6, 2019; Accepted: November 23, 2019; Published: December 6, 2019

\begin{abstract}
In Quantum Mechanics, one knows that the wave function interpretation is probabilistic. We previously established that any particle scalar field is the cause of its existence. Here, one examined the plane solution regarding a moving particle in vacuum, through the relativistic formalism. It appeared the following. (i) The solution presents four alternatives, like in Dirac unified formalism; when searching stationary solutions of the system vacuum-particle or the system vacuumantiparticle. (ii) Considering the former, each spinner component shows the interaction of one particle charge with three vacuum fermions of spin- $1 / 2$; each oriented along one space direction. Furthermore, this allows deducting the triple nature of any gauge fermion. (iii) Each solution case is definable with a same wave front width. This determination became possible from the vector companion of that wave function one introduced before. Here, this points out the existence of transverse time. (iv) Both functions let emphasizing the existence of a third fundamental field of long range, which is identifiable to the fundamental spin field. $(v)$ This unites the particle spin and orbital momenta and bears in addition a magnetic-like field, which is yet unknown. (vi) According to the charge, a particle field is observable in wave phenomena, from the manifestations of its gauge fermions or gauge bosons; when ejected from their stationary states by a perturbation... At last, the results highlight the quantum composition of wave functions, the spin-field patency, and the wave nature manifestation from five differentiable fields.
\end{abstract}

Keywords: Duality Field-Matter, Klein-Gordon Equation, Quantum Mechanics, Quantum Vacuum, Spin Field, Transverse Time, Wave Front, Wave Function, Wave Nature

\section{Introduction}

The wave function of Schrodinger equation became effective since the dawn of Quantum Mechanics. It is the sole uniting the wave nature of particles, whatever the tested field is (see [1, 2]). This explains its importance in modern physics, owing to its remarkable predictions (see [3-5]). Nevertheless, its probabilistic interpretation hides the physical reality one can expect to find out from the simplest case of a free particle. The same function appears in KleinGordon equation, which takes count of the relativistic relation energy-momentum $[6,7]$. The equation has the form of any classical wave equation (see [8]). This should suggest that the related wave must necessarily correspond to a physical field. In classical physics, the elastic field propagation is often understandable from successive elastic shocks between unit cells of the host medium. One should take count of this reality since this is the seat of wave propagation. One can understand that such a process did not seem applicable at the time, without recalling the former and hypothetical existence of the aether. For some reasons however, P. A. M. Dirac established a more adapted equation for a wave function having four components [9-11]. The equation solution brought out the existence of fermions and antifermions. These lasts permitted to foresee antimatter existence for the first time. This result suggested reconsidering the aether existence at the new light $[12,13]$. Nowadays, this is surely what one can call "quantum vacuum". In this context, Dirac fermions should represent vacuum unit cells. However, one does not yet know the relatable field.

From both field equations of Duality Field-Matter [14], such fermions appear too. They are describable by the components of both scalar and vector gauge fields satisfying 
these equations. This allows obtaining four gauge couplings. The differentiation conditions of fields at cell interfaces, in the model of vacuum lattice, led to identifying the common four fundamental fields. Because of this, we qualified the original field by the unified field. In the duality occurrences [15], it already appeared that each differentiated field originates a couple of scalar and vector wave functions. In addition, a fifth gauge coupling appeared which suggests the existence of a fifth fundamental field. In this paper, one then aims to emphasize this existence and the nature of Dirac's fermions from both wave functions exploration.

Hence, it will be useful to examine the evolution stages of the scalar wave function, while researching to complete the missing information. Then one will discuss the approach consequences.

\section{Notable Occurrences in Quantum Mechanics}

One considers here a perfect particle of mass $m$ that moves freely at the speed $\vec{v}$ along abscissa axis in quantum vacuum. The method here consists in reexamining the wave function solutions from the classical formalism to the relativistic one, while applying the elementary mathematics rigor emphasizing physical events.

\subsection{In Classical Formalism: Information Loss}

The Hamiltonian and impulse are the main operators in Quantum Mechanics. These originate quantization in linked systems. If the vector column $(\vec{r}(x, y, z), i c t)^{\dagger}$ designates the spacetime position in the particle atmosphere, they respectively read.

$$
H=i \hbar \frac{\partial}{\partial t} ; P=-i \hbar \vec{\nabla}
$$

If $E$ is the particle energy and $\psi(\vec{r}, t)$ is the wave function that describes vacuum perturbations, one writes the equation

$$
H \psi=E \psi
$$

This solution is $\psi(\vec{r}, t)=u(\vec{r}) \exp (-i E t / \hbar)$. For a free particle of momentum $\vec{p}\left(p_{x}, p_{y}, p_{z}\right)$, one has in general

$$
P \psi=\vec{p} \psi
$$

That is

$$
\left\{\begin{aligned}
-\frac{i}{\hbar} \frac{\partial}{\partial x} u(\vec{r}) & =p_{x} u(\vec{r}) \\
-\frac{i}{\hbar} \frac{\partial}{\partial y} u(\vec{r}) & =p_{y} u(\vec{r}) \\
-\frac{i}{\hbar} \frac{\partial}{\partial z} u(\vec{r}) & =p_{z} u(\vec{r})
\end{aligned}\right.
$$

From the method of variable separation, this yields the determination of $u(\vec{r})$ and one finally obtains

$$
\psi(\vec{r}, t)=u_{0} e^{-\frac{i}{\hbar}(E t-\vec{p} \vec{r})} ; u_{0} \in \mathbb{C}
$$

This corresponds to the simplest plane wave. In $x$ - direction, one has $\vec{p} \vec{r}=p_{x} x$. Note hence that the second member does no longer depend on the variables $y$ and $z$. There is information loss in both directions transverse to the motion. Consequently, the operator definitions are surely incomplete!

\subsection{In Relativistic Formalism}

By calculating the quantity $\left(H^{2}-P^{2}\right) \psi$, one obtains Klein-Gordon equation from energy-momentum relation, i.e.

$$
\left(\Delta-\frac{1}{c^{2}} \frac{\partial^{2}}{\partial t^{2}}\right) \psi=\left(\frac{m c}{\hbar}\right)^{2} \psi
$$

This classical analysis allows completing the previous formalism, as one can expect. One can also remark that the relation of energy-momentum is not valid only for elementary particles; Special Relativity does not imply such a constraint. That is, the action quantum could be different. In Duality Field-Matter indeed, one showed that both usual operators (1) are redefinable to near a constant. In that equation, this comes to substituting the particle real mass by an effective mass; which can be different than the former. One implies this here.

\subsubsection{Information Gain}

Remark that the previous equation also obtains with the opposite operators, such that, in principle, one must consider the definitions

$$
H= \pm i \hbar \frac{\partial}{\partial t} ; P=\mp i \hbar \vec{\nabla}
$$

which take count of antiparticle existence (sign - in $H$ ). This means that the wave equation is valid for four combinations of operators. The four possible wave functions could have suggested Dirac to linearize the previous equation in four dimensions.

With respect to the motion direction $(O x)$, that equation becomes equivalent to the system

$$
\left\{\begin{array}{c}
\left(\frac{\partial^{2}}{\partial x^{2}}-\frac{1}{c^{2}} \frac{\partial^{2}}{\partial t^{2}}\right) \psi=\left(\frac{m c}{\hbar}\right)^{2} \psi \\
\left(\frac{\partial^{2}}{\partial y^{2}}+\frac{\partial^{2}}{\partial z^{2}}\right) \psi=0
\end{array}\right.
$$

One often forgets the second one, perhaps for simplification. Here, the solution must combine progressive and regressive components. For the Hamiltonian of the particle defined with the positive sign, this takes the form

$$
\begin{gathered}
\psi(\vec{r}, t)=u(y, z)\left(\beta_{+} e^{i k x}+\beta_{-} e^{-i k x}\right) \mathrm{e}^{-\mathrm{i} \omega t} ; \\
\omega=\frac{E}{\hbar} ; k=\frac{p_{x}}{\hbar}
\end{gathered}
$$

The wave propagates in one sense or another at de Broglie celerity $c_{B}=\omega / k$ i.e. $c_{B}=c^{2} / v$. If the progressive component was expectable from the classical formalism, the regressive one was not. However, this can become manifest in particle backward emissions, e.g. during the so-called braking radiation, when an electron passes close to a nucleus. That solution is then inevitable. After substituting the expression in the second equation of (7), the method of 
variation separation allows setting $u(y, z)=u_{y}(y) \cdot u_{z}(z)$, such as one gets

$$
\frac{\partial^{2} u_{y}}{\partial y^{2}}+\frac{\partial^{2} u_{z}}{\partial z^{2}}=0
$$

Thence it suits deducting that

$$
\frac{\partial^{2} u_{y}}{\partial y^{2}}=\alpha ; \frac{\partial^{2} u_{z}}{\partial z^{2}}=-\alpha ; \alpha \in \mathbb{C}
$$

After integrations, the solutions can write under the forms

$$
\left\{\begin{array}{l}
u_{y}(y)=+\alpha\left[\left(y-y_{0}\right)^{2}+\delta_{y}\right] \\
u_{z}(z)=-\alpha\left[\left(z-z_{0}\right)^{2}-\delta_{z}\right]
\end{array}\right.
$$

For a perfect particle, space is homogenous around. One must have $u_{y}(y)=u_{z}(z)$. One gets the circle equation of center $\left(y_{0}, z_{0}\right)$ and radius $R$, such as

$$
\left\{\begin{array}{c}
\left(y-y_{0}\right)^{2}+\left(z-z_{0}\right)^{2}=R^{2} \\
R=\sqrt{\delta_{z}-\delta_{y}}
\end{array}\right.
$$

This lets writing

$$
y(\theta)-\mathrm{y}_{0}=R \cdot \cos (\theta) ; z(\theta)-\mathrm{z}_{0}=R \cdot \sin (\theta)
$$

where in $\theta$ is a parameter, which cannot depend on the usual time $t$ (see next section). To reduce the number of integration constants to the necessary ones, it is enough to note the dependence of both constants defining the radius; the origin consideration as well. One must then set

$$
y_{0}=0 ; z_{0}=0 ; \delta_{y}=0 \Rightarrow \delta_{z}=R^{2}
$$

The solution (8) finally reads

$$
\psi(\vec{r}, t)=\alpha^{2} y^{2}\left(R^{2}-z^{2}\right)\left(\beta_{+} e^{i k x}+\beta_{-} e^{-i k x}\right) \mathrm{e}^{-i \omega t}
$$

with $y^{2}+z^{2}=R^{2}$. There are three remaining integration constants, accordingly to the number permitted by the system of both differential equations. The wave front width is $2 R$. It will appear that the radius is equal to the wavelength. In practice, a wider wave front implies some harmonics of the most general solution; that given here corresponds to the fundamental one.

\subsubsection{Wave Function Having Components}

Given that the particle must not radiate energy so that its motion remain uniform, its suits considering stationary solutions. One obtains these for both conditions

$$
\beta_{-}=+\beta_{+} \text {or } \beta_{-}=-\beta_{+}
$$

Since both cases are independent, the pure solution is translatable by the vector

$$
\left(\begin{array}{l}
\psi_{+}(\vec{r}, t) \\
\psi_{-}(\vec{r}, t)
\end{array}\right)=y^{4}\left(\begin{array}{c}
a \cdot \alpha^{2} \cos (k x) \\
i b \cdot \alpha^{2} \sin (k x)
\end{array}\right) \mathrm{e}^{-\mathrm{i} \omega t}
$$

The first member represents the particle state in Dirac sense; expressible in an abstract base of Hilbert space. Each component is relatable to the probability to find the particle inside a given interval of abscissas. If necessary, one should find that the amplitudes go to zero with the interval extension. For illustration, Figure 1 shows for instance the relative amplitude of the second component. One can remark that the particle must be inside the cylinder defined by the oscillating amplitude envelope, whose diameter is the wave front width.
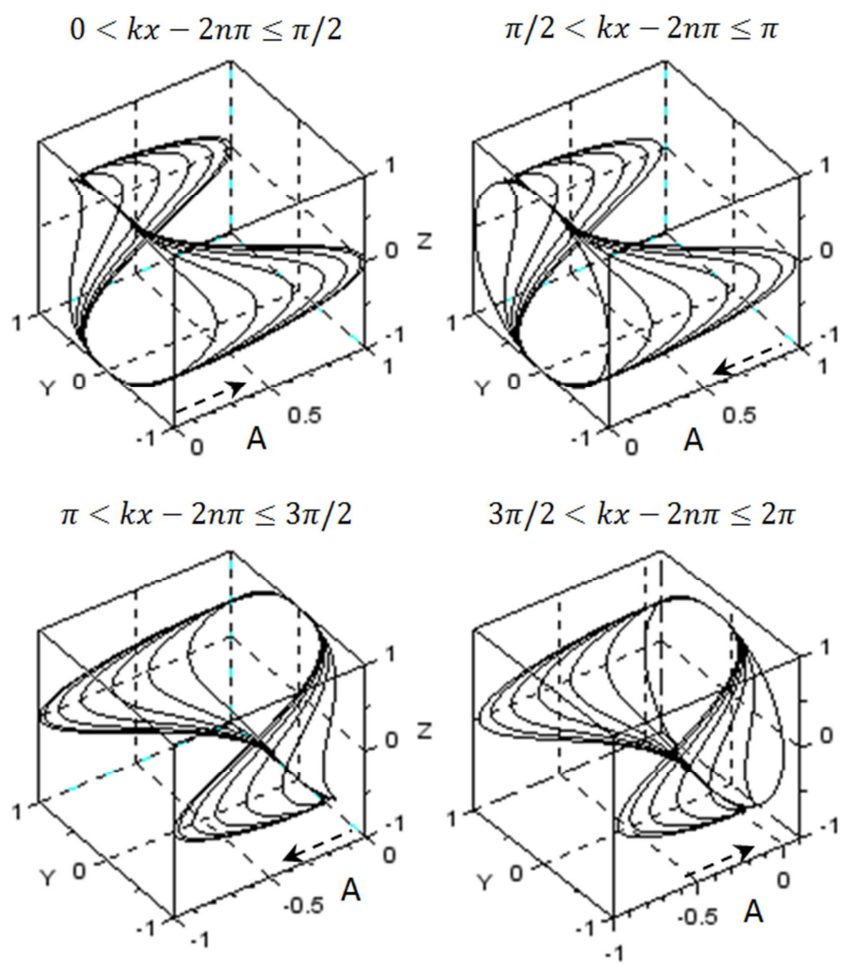

Figure 1. Amplitude of the wave function component $\psi_{-}(\vec{r}, t)$ in space: in each case (see $k x$ interval $\forall n \in \mathbb{N}$ ), the set of curves try to indicate the amplitude (A) envelope. The projection of curves on the plane (Oyz) is a circle (see $2^{\text {nd }}$ and $4^{\text {th }}$ cases). The different curves are symmetric with respect to $y$ and $z$. These 4-dimension representation shows the way the amplitudes vary on one space period, i.e. one wavelength; the arrows indicate these increasing and decreasing, about the function $\sin (k x)$.

In addition, note now that the Hamiltonian of the relatable antiparticle, defined with the negative sign, also produces two analogous solutions into $(+i \omega t)$. The four solutions are then relatable to Dirac wave function in his unified formalism. As one already knows, what is findable with the particle is deductible for its opposite.

\subsubsection{Wave Function as Spinner}

When applying the stationariness conditions above, both independent solutions can write under the initial form of spinner

$$
\left(\begin{array}{l}
\psi_{+}(\vec{r}, t) \\
\psi_{-}(\vec{r}, t)
\end{array}\right)=\alpha^{2} \mathrm{y}^{4} \underbrace{\left(\begin{array}{cc}
a & a \\
b & -b
\end{array}\right)}_{\sigma}\left(\begin{array}{l}
\mathrm{e}^{-\mathrm{i}(\omega t-k x)} \\
\mathrm{e}^{-\mathrm{i}(\omega t+k x)}
\end{array}\right)
$$

The indicated matrix is writable as the following linear combination of Pauli's matrixes $\sigma_{i}$ and the identity matrix II , such as

$$
\sigma=\frac{a-b}{2}\left(i \sigma_{x}+\mathbb{I}\right)+\frac{a+b}{2}\left(\sigma_{y}+\sigma_{z}\right)
$$


Here, one has to choose the axis orientations such as

$$
\sigma_{x}=\left(\begin{array}{cc}
0 & -i \\
i & 0
\end{array}\right), \sigma_{y}=\left(\begin{array}{cc}
1 & 0 \\
0 & -1
\end{array}\right) ; \sigma_{z}=\left(\begin{array}{cc}
0 & 1 \\
1 & 0
\end{array}\right)
$$

These matrixes correspond to fermions- $1 / 2$ relatively to both possible orientations of each. Such fermions can only constitute vacuum unit cells mentioned in the introduction, while II identifies the particle (see result section). In the expression of $\sigma$ in addition, the matrix combinations are striking. These express the fact that the interaction is different in the motion direction and in both perpendicular directions. Like in material media, vacuum must be submitted to compression stresses and to shear stresses respectively. This implies the existence of both longitudinal and transverse fields (see below); manifestly, the latter must originate the wave front. Consequently, it is deductible that one must physically have $(b \neq \pm a)$ in general.

Because of this and in order to reduce the number of integration constants (see (17)), one can set

$$
\left\{\begin{array} { l } 
{ \frac { a - b } { 2 } = \operatorname { s i n } ^ { 2 } ( \varphi ) } \\
{ \frac { a + b } { 2 } = \operatorname { c o s } ^ { 2 } ( \varphi ) }
\end{array} \Rightarrow \left\{\begin{array}{l}
a=1 \\
b=\cos (2 \varphi)
\end{array}\right.\right.
$$

When assuming that $\varphi \in[0, \pi]$ in complement to the angle $\theta$ above, one can infer that both angles would define an angular quantity in spherical coordinates. With regard to the spin implication here, this should match the particle angular momentum (see result section).

Moreover, inasmuch as there is no privilege direction for such a vector, it is expectable that this rotates in course of time. That is, both angles should vary in function of a nonstandard time (see below). However, because of $a$ value, the second angle only implies the component $\psi_{-}(\vec{r}, t)$, which is more probable. This is the microscopy of the scalar wave function.

\section{Other Dualistic Occurrences}

This section completes the previous formalism in allowing determining the wave front radius, from the wave function companion already notified. Then it deals with both functions connection. Here, the origin of that companion is introducible otherwise for continuity reasons.

From the general solution of plane waves indeed (see for instance (4)), one can define the wave planes by $(E t-\vec{p} \vec{r}) /$ $\hbar=2 \pi q ; \forall q \in \mathbb{Z}$. When taking the differentials of both members, one gets to

$$
\vec{p} \frac{d \vec{r}}{d t}=E
$$

Along the motion direction $(O x)$, de Broglie celerity defines by $c_{B}=d x / d t$, such as $p_{x} c_{B}=E$. Both other derivatives being non-zero, a transverse celerity should exist (see spinner solution above). This must match with the existence of vector wave function. Figure 2 shows both relatable transverse waves, with respect to the longitudinal one.
Given that both wave functions must be independent in behavior (they must respect different equations), the last one is definable with a new spacetime. This must only differ from the first, considered by default, through transverse time. The transverse space coordinates must however be identical to the longitudinal ones, owing to both functions coexistence (see below).

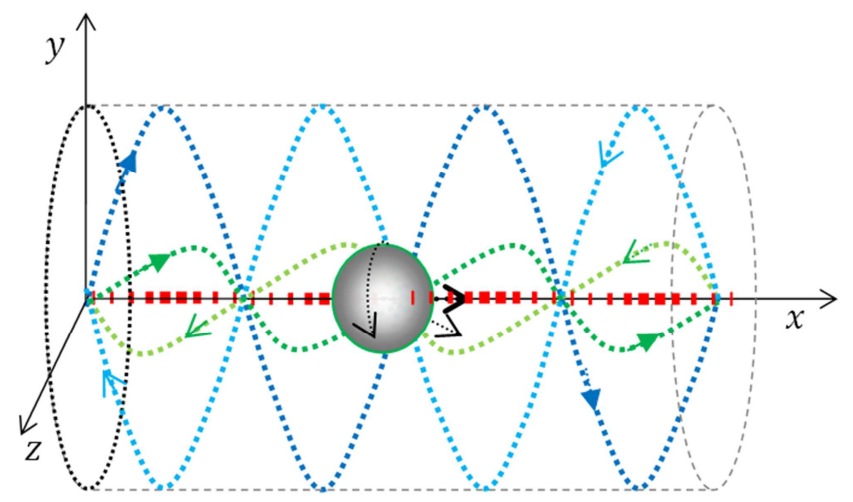

Figure 2. Three kinds of wave propagation along $x$-direction: the sphere represents the particle at a given position; its size is only illustrative and the associated arrows try to indicate the resulting vector between the spin momentum (horizontal) and the orbital one (transverse), foreseeable from the wave function microscopy. The short vertical lines represent the case of one longitudinal wave. The vertical or horizontal sinusoids delimit the cases of transverse waves. The arrows indicate the possible propagation senses in interference case.

\subsection{De Broglie-like Transverse Celerity}

With regard to the deductions (13) excluding the default time, one has to assume the existence of transverse time $t$, such as

$$
d y / d t^{\prime}=-R \dot{\theta} \sin (\theta) ; d z / d t^{\prime}=R \dot{\theta} \cos (\theta)
$$

This defines the celerity of module

$$
c_{m}=R \dot{\theta}
$$

On the other side, this is certainly the one already emphasized by Duality Field-Matter for the field-theorem demonstration. It is relatable to any particle charge and is definable by the ratio between the kinetic energy $(T=$ $\left.m c^{2}(\gamma-1)\right)$ and the momentum $\left(p_{x}=\gamma m v\right)$, i.e.

$$
c_{m}=\frac{c^{2}}{v}\left(1-\frac{1}{\gamma}\right)
$$

Contrarily to de Broglie wave packet which corresponds to a normal dispersion $\left(v<c_{B}\right)$, the corresponding wave packet corresponds to an abnormal dispersion $\left(v>c_{m}\right)$, which can imply other transverse components. For photons or gravitons $\left(v=c \Rightarrow c_{m}, c_{B}=c\right)$, there is no dispersion while the wave front exist!

\subsection{Wave Front Radius}

Since the related motions of vacuum fermions is not always on a plane but unrolls in course of transverse time, the relation (23) has no connection with a plane motion; the 
speed $\dot{\theta}$ is then interpretable as the frequency with regard to the particle kinetic energy. It suits putting $T=h \dot{\theta}$; where $h$ is Planck's constant. With the previous definition of $c_{m}$, one then deducts that

$$
R=\frac{2 \pi}{k}
$$

That is, the wave front radius is equal to the wavelength (see solution (15)).

\subsection{Connection Between Wave Functions}

If $\vec{\chi}\left(\vec{r}, t_{t}\right)$ designates the wave function companion of $\psi$, one already showed that both form the 4 -vector $(\vec{\chi}, i \psi / c)$, at cell interfaces of vacuum lattice, where occur differentiations (see relation (10) in [15]); both relatable gauge fields are there proportional and one should have here $t^{\prime} \equiv t$. With adaptation of the former relations to any particle (substitution of $c$ by $c_{B}$ ), this vector then respects the gauge relations

$$
\left\{\begin{array} { c } 
{ c _ { B } ^ { 2 } \vec { \nabla } \vec { \chi } = \eta \partial _ { t } \psi } \\
{ \partial _ { t } \vec { \chi } = \eta \vec { \nabla } \psi }
\end{array} \text { with } \left\{\begin{array}{c}
\partial_{\mathrm{t}}=\partial / \partial \mathrm{t} \\
\eta \neq \pm 1
\end{array}\right.\right.
$$

The special case where $\eta=+1$ corresponds to gravitation while that of $\eta=-1$ is to electromagnetism; Lorentz's gauge appears besides for the electric charge conservation. When substituting $\psi$ by the spinner solution, one obtains a vector spinner solution. From the second equation, it appears that $\psi$ composition is also that of each $\vec{\chi}$ component. By taking in addition the suitable derivatives of either equation in time or space then subtracting the results, one gets to the equation system

$$
\left\{\begin{array}{l}
\square \psi=0 \\
\square \vec{\chi}=\overrightarrow{0} ; \square=\Delta-\frac{1}{c_{B}^{2}} \frac{\partial^{2}}{\partial t^{2}}
\end{array}\right.
$$

A similar result is obtainable with the differentiations of both gravitational and electromagnetic fields (see Table 2 in [16]). These are equivalent to Klein-Gordon equation for stationary solutions. Because of this new differentiation, there exists a third fundamental field of long range; if $\eta$ is unique (see result section).

\subsection{Extending Gauge Coupling in the Current Duality}

Out of cell interfaces however, the complete equations of both previous quantities are derivable from the gauge relations of Duality Field-Matter [14]. These become generalizable by substituting the alternative \pm by $\pm \eta$, at the end of a procedure of gauge obtainment; in order to take count of the new differentiation; one could introduce this before but the reason did not seem obvious at the time. One must then have the following relations in gauge fields $\left.A_{S}\right\rangle=$ $\left(\vec{A}_{s}, i V_{s} / c\right)$ and $\left.A_{v}\right\rangle=\left(\vec{A}_{v}, i V_{v} / c_{B}\right)$.

$$
\left\{\begin{array}{c}
\partial_{\mathrm{t}} \vec{A}_{s} \pm \eta \vec{\nabla} V_{s}=\overrightarrow{0} \\
\vec{\nabla} \vec{A}_{v} \pm \eta \partial_{t^{\prime}} V_{v} / \mathrm{c}_{\mathrm{B}}^{2}=0
\end{array} ; \eta \in \mathbb{C}^{*}\right.
$$

These combinations define from now on five fundamental fields. One can show that the choice of two different coefficients $\eta$ does not match a free particle case. Therefrom, it suits setting the relations

$$
\left\{\begin{array}{c}
\vec{A}_{s}=\mp \eta \beta_{s} \vec{\nabla} \psi \\
V_{s}=+\beta_{s} \partial_{t} \psi
\end{array} ;\left\{\begin{array}{c}
\vec{A}_{v}=\mp \eta \beta_{v} \partial_{t} \vec{\chi} \\
V_{v}=+\beta_{v} c_{\mathrm{B}}^{2} \vec{\nabla} \vec{\chi}
\end{array} ; \beta_{\mu} \in \mathbb{C}^{*}\right.\right.
$$

These show for any field that the scalar gauge field $(s)$ is the cause of the scalar wave function variations in spacetime; the vector one $(v)$ is that of its companion variations. One can see that in spinner representation, one obtains two possible expressions of each component of gauge field. Each one must correspond to either spin-1/2 projection of related fermions.

\section{Results and Discussion}

This section brings out the happenings of previous sections. It screens the relatable consequences, with regard to the particle field-nature alias its wave nature.

\subsection{General Occurrences}

One determined the expression of the scalar wave function along with its front as detectable in experiments (see subsubsection 2.2.1). When considering a non-radiating particle in vacuum, one obtained Dirac-like solution in stationary waves (see sub-subsection 2.2.2). The four possible components are separable into two of the test particle and two others of this antiparticle. Regarding the particle itself, the relatable spinner allows understanding the microscopy of that function.

From the expression (19) indeed, Pauli's matrices must necessarily represent vacuum fermions, such as these correspond to vacuum excitation at the particle presence (see identity operator). For instance, if one considers that the particle is electrically charged, the identity operator should represent this characteristic. It would excite vacuum in the motion direction, which spontaneous generate three photon fermions; each oriented along one space direction. Hence, the operator identity must indicate one of the particle characteristics. The causalities defined by the relations (29) sustain this and show that the scalar gauge field $\left(\vec{A}_{s}, i V_{s} / c\right)$ of the particle originates the wave function. Each spinner component then defines either a fermion or an antifermion. This applies in both perpendicular directions too (see $\vec{\chi}$ meaning from (26)). Thence, note that either particle is finally a triplet of vacuum fermions. Certainly, this reveals the enigma origin of gauge-fermion triplicity.

\subsection{The Fifth Fundamental Field}

One also showed that a wave front width of the scalar wave function is determinable when emphasizing otherwise its vector companion (see subsection 3.2). This lets deducting the existence of a new field of long range (see subsection 3.3). To identify this, one can refer to the missing fundamental characteristic of particles in fundamental field, i.e. the spin. Indeed, one knows that a particle can have an orbital momentum, in addition to its intrinsic spin (see [17, 18]). This is transverse to the motion direction and is then 
linkable here to the vector wave function, or better to the vector gauge field $\left|A_{v}\right\rangle$; the preceding one being to the scalar gauge field $\left|A_{s}\right\rangle$ (see above).

Indeed, contrarily to both other fields of long range $(\eta=1)$, $\left|A_{s}\right\rangle$ admits an electric-like field, which is definable by the expression below; the signs correspond to those of the deductions (29).

$$
\vec{E}_{s}=-\vec{\nabla} V_{s} \mp \partial_{t} \vec{A}_{s} \Rightarrow \vec{E}_{s}=-\beta_{s}(1-\eta) \partial_{t} \vec{\nabla} \psi
$$

The magnetic-like field $\vec{B}_{s}=\vec{\nabla} \times \vec{A}_{s}$ is zero. It suits defining $\vec{E}_{s}$ as the spin momentum, i.e. such as $\left[\mathrm{E}_{s}\right]=[\hbar]$. The orbital momentum must as well be definable from the 4 vector of $\vec{A}_{v}$ such that $\left[\mathrm{E}_{v}\right]=[\hbar]$. The magnetic-like field $\vec{B}_{v}=\vec{\nabla} \times \vec{A}_{v}$ exists of course. This originates the particle rotation, which attests an internal activity; therefore its eventual compositeness. This sustains the triplicity of fermions indicated above. In addition, this vector unit is $\left[B_{v}\right]=[$ mass $][$ distance $]$ and is understandable as originated in time by a transverse momentum. Until now, one does not yet know this transverse quantity. However, this surely justifies the adopted concept of spin, postulated early by Pauli. One can hence infer that the new differentiation certainly corresponds to the spin field.

Moreover, this regards, not only the indicated vector, but also the dualistic scalar fields given by, in regard to the same deductions.

$$
\left\{\begin{array} { l } 
{ \Gamma _ { s } = \pm \vec { \nabla } \vec { A } _ { s } + \frac { 1 } { c ^ { 2 } } \partial _ { t } V _ { s } } \\
{ \Gamma _ { v } = \pm \vec { \nabla } \vec { A } _ { v } + \frac { 1 } { c _ { B } ^ { 2 } } \partial _ { t ^ { \prime } } V _ { v } }
\end{array} \Rightarrow \left\{\begin{array}{l}
\Gamma_{s}=\beta_{s}\left(-\eta \Delta \psi+\frac{1}{c^{2}} \partial_{t}^{2} \psi\right) \\
\Gamma_{v}=\beta_{v}(-\eta+1) \partial_{t^{\prime}}(\vec{\nabla} \vec{\chi})
\end{array}\right.\right.
$$

The second one $\left(\Gamma_{v}\right)$ is deductible by analogy from the first in transverse time $t$ ' one perhaps does not yet know in common theories. The spin field has then more components than the other fields, because of the coefficient $\eta$ to be determined. Its scalar behavior is surely that remarkable in anisotropic media as spin waves one knows since long. However, the concept of fundamental spin field does not appear in other quantum field theories, one already knows incomplete, though remarkable in many other works (see [19-21]). Here, its notable existence sounds theoretically explicit. In absence of other characteristics, this is surely the first subtle field, which precedes the manifestations of gravitational, electromagnetic, weak and strong fields. For instance, it allows understanding that the particle proper rotation must originate the first field.

\subsection{Manifested Particles in Wave Phenomena}

Recall now that though the particle field nature, explainable by the wave function, comes from the sole scalar gauge field, the transverse one is implied. That is, both scalar and vector gauge fermions, or their host bosons, must be observable in experiments, e.g. from the diffraction phenomenon through a hole. Accordingly to the dualistic gauge couplings of fermions/antifermions, gravitons are couples $(-,-)$, photons are couples $(+,+)$, weak bosons are couples $(-,+)$ and strong bosons are couples $(+,-)$. Hence, the complete description of the wave nature must imply a specific charge. From the wave front content, the manifestable particles are then either gauge fermions or gauge bosons. It sounds obvious that the formers are only possible if the field originating the phenomenon is instable, like the weak or strong field.

In the current duality, those sub-particles are fundamental if the particle speed $v$ is equal to that of light $c$ (case of photons); otherwise, they are non-fundamental. Their manifestations are only understandable when they go out of their brief stationary states, after the wave front perturbation. With an electron for instance, massive photons must produce a diffraction image, if this particle electromagnetism was disturbed. As well, non-fundamental weak bosons must produce such an image, if the electron weak field was disturbed. However, given that weak bosons are instable, one could observe, after these decays, a diffraction image in the motion direction and surely fermion emission in the perpendicular direction! The first event should come from scalar fermions, which are non-fundamental antileptons. The second should come from vector fermions, which are nonfundamental leptons. It is also expectable that the particle wave nature be observable from spin bosons; which are surely stable, like those of gravitation or electromagnetism. This is surely the case, when any other field is credible.

\section{Conclusion}

From a free particle in motion, one showed that the relativistic wave function emphasizes enough information. The stationary solution of the system particle-vacuum allows deducting four Dirac-like solutions; two regard the particle while two others regard its antiparticle. Each alternative component of spinner represents the particle interaction with three fermions of quantum vacuum, owing to the three Pauli's matrices in the solution. However, the complete determination of the common wave front width required considering the vector companion of the scalar wave function. This represents the transverse interaction with quantum vacuum in transverse time. In addition, both functions express a new differentiation of gauge coupling, which implies an additional field of long range.

By examining the result, it also appeared the following. ( $i)$ The particle interacts with vacuum via its fields or charges. This medium reacts accordingly to each by fermion triplets, oriented in the three space directions; these must surely oscillate. (ii) Such fermions are surely those of the unified field, which structures quantum vacuum; this governs both field equations of Duality Field-Matter. (iii) Furthermore, it appears that each scalar or vector gauge fermion of the particle field originates these vacuum fermions. This allows deducting the triplicity origin of any manifested gauge fermion; this constituted an enigma in physics. (iv) That new differentiation corresponds to the spin field. Both intrinsic spin and orbital momenta of any particle match electric-like fields. (v) They have a magnetic-like companion one does not yet know! This sustains in addition the existence of transverse time. ( $v i)$ The complete description of the particle 
wave nature is observable through manifestations of gauge fermions or gauge bosons of disturbed field. The formers should be manifest with instable fields.

This study completed the previous one on the physical origin of wave functions. It raised the number of fundamental fields to five, with regard to the known characteristics of particles. However, it seems that another fundamental field of long range may exist! These are our findings.

\section{References}

[1] G. Matteucci (2013). Interference with electrons - from thought to real experiments. Proc. Of SPIE 8785, 8785CF-1.

[2] P. Grangier, G. Roger and A. Aspect (1986). Experimental evidence for a photon anti-correlation effect on a beam splitter: A new light on single-photon interferences. Europhys. Lett 1, 173-179.

[3] I. V. Kanatchikov (2018). The solution of Schrodinger equation justifies early Bohr atomic model. Rep. Math. Phys. 82,373 .

[4] S. Zeller (2018). Determination of the He-He, Ne-Ne, Ar-Ar, and $\mathrm{H} 2$ interaction potential by wave function imaging. Phys. Rev. Lett. 121, 083002.

[5] S. P. de Alwis (2019). The Wave Function of the Universe and CMB Fluctuations. Phys. Rev. D 100, 043544.

[6] Iveta Semoradova (2017). Crypto-Hermitian Approach to the Klein-Gordon equation.' Acta Polytechnica 57, 462-466.

[7] E. J. A. Curi, L. B. Castro and A. S. De Castro (2019). Proper treatment of scalar and vector exponential potentials in the Klein-Gordon equation: Scattering and bound states. Eur. Phys. J. Plus 134, 248.

[8] L M. Moukala (2018). Characterization of cubic crystalline systems: a field theory uniting elasticity and electromagnetism. Res. J. Material Sci. 6, 1-4.
[9] P. A. M. Dirac (1928). The Quantum Theory of the Electron. Proceedings of the Royal Society of London. Series A 117, 610-624. http://www.jstor.org

[10] L. Alvarez-Gaumé, M. A. Vazquez-Mozo (2012). An invitation to quantum field theory. Lectures Notes in Physics 839.

[11] M. Mansuripur (2019). Spin-orbit coupling in the hydrogen atom, the Thomas precession, and the exact solution of Dirac's equation. Spintronics XII, Proceedings of SPIE 11090, 110901X.

[12] P. Dirac, (1951). Is there an aether?. Nature 168, 906.

[13] N. C. Petroni, J. P. Vigier (1983). Dirac's aether in relativistic quantum mechanics. Foundations of Physics. 13, 253.

[14] L. M. Moukala (2018). Ultimate duality field-matter: fields structural unification. Res. Recent Sci. 7, 1-9.

[15] L. M. Moukala (2019). Duality occurrences: physical origin of waves functions. International Journal of Applied Mathematics and Theoretical Physics 5, 15-19.

[16] L. M. Moukala (2017). The unified energy as vacuum quintessence in wave equations. Res. J. Physical Sci., 5, 1-6.

[17] M. Mansuripur (2011). Spin and orbital angular Momenta of electromagnetic waves in free space. Physical Review A 84, 033838 .

[18] K. Y. Bliokh, M. R. Dennis, F. Nori (2017). Position, spin and orbital angular momentum of a relativistic electron. Phys. Rev. A 96, 023622.

[19] X. Bekaert, Mourad and M. Najafizadeh (2017). Continuousspin field propagator and interaction with matter. JHEP 1711, 113.

[20] D. Sorokin and M. Tsulaia (2018). Higher spin fields in hyperspace: a review. Universe 4, 7.

[21] K. Benakli, Y. Chen, P. Cheng and G. Lafforgue-Marmet (2019). Stochastic gravitational waves from spin-3/2 fields Hunting SUSY in the sky. Phys. Rev. D 99, 095032. 